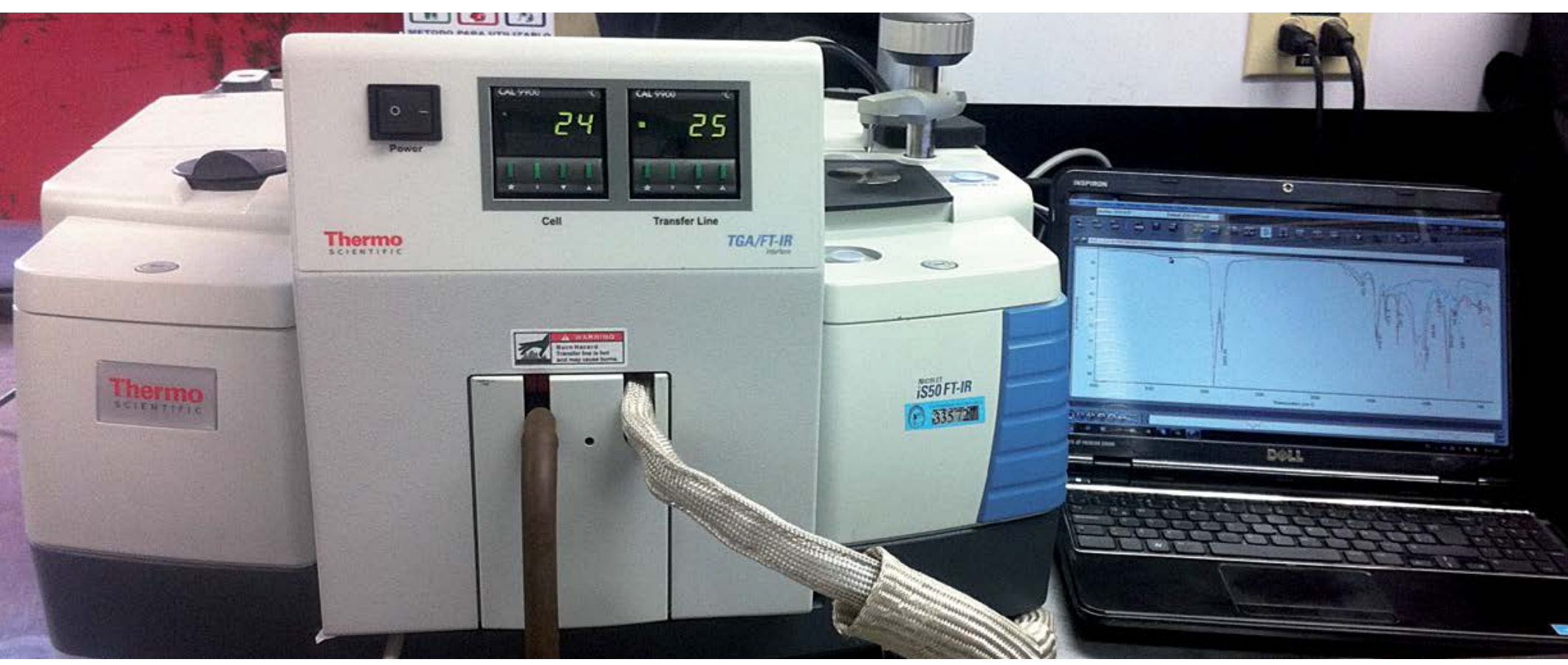

\title{
Métodos de ensayo para la determinación cualitativa y cuantitativa del contenido de polímero presente en ligantes asfálticos modificados
}

Fecha de recepción: 32 de setiembre de 2013

Fecha de aceptación: 10 de octubre de 2013

\section{Resumen}

En Costa Rica, como en muchos países, se está implementando el uso de asfaltos modificados con polímeros en la construcción de carreteras, el mercado creciente ofrece varios tipos de polímeros que mejoran notablemente las propiedades reológicas de los asfaltos, comúnmente del tipo SBR, SBS, polvo de hule de llanta, EMA, EVA entre otros. Los porcentajes de modificación con respecto al ligante asfáltico están entre el $1 \%$ al $5 \%$ $\mathrm{m} / \mathrm{m}$. Sin embargo, a pesar del hecho anterior, poca investigación se ha realizado en la cuantificación de la cantidad de polímero incorporado en el ligante asfáltico.

En consecuencia, el presente estudio pretende revisar los métodos para determinar, de forma cuantitativa y cualitativa, la presencia de polímeros presentes en el ligante asfáltico. Este artículo está dirigido principalmente a los laboratorios de ensayo que ejecutan ensayos a ligantes asfálticos y sus derivados, pero también a los ingenieros y profesionales que trabajan en la construcción de obras viales y en su control de calidad.
Quim. Jorge Salazar Delgado,

Unidad de Materiales y Pavimentos, LanammeUCR

Tel.: (506) 2511-4963 / E-mail: jorge.salazardelgado@ucr.ac.cr

\section{Abstract}

Costa Rica, as many countries, are implementing the use of polymer-modified asphalts for road construction, the growing market offers several types of polymers that improve the rheological properties of asphalts, significantly. The most common polymers are SBR, SBS, tire rubber in powder, EMA and EVA among others. The modifiers percentages are between $1 \%$ to $5 \% \mathrm{~m} / \mathrm{m}$ of the asphalt binder. However, little research has been conducted for quantifying the amount of polymer incorporated into the asphalt binder.

Accordingly, the methods to determine qualitatively or quantitatively the presence of polymers present in the asphalt are the objective of this. This article is aimed to asphalt binder's laboratories, mainly, but also to engineers and professionals working on road construction and Quality Assurance.

Keywords: Asphalt, bitumen, asphalt binders, polymer, SBS, SBR, FTIR, IR. 


\section{Introducción}

El asfalto ha sido utilizado para la construcción de carreteras desde la antigüedad ya que tiene ciertas características físicas que le permiten su uso como aglutinante en la mezcla asfáltica. Como el asfalto es un material altamente impermeable, adherente y cohesivo, capaz de resistir altos esfuerzos instantáneos y fluir bajo la acción de cargas permanentes, presenta las propiedades ideales para la construcción de pavimentos cumpliendo las siguientes funciones:

- Impermeabilizar la estructura del pavimento, haciéndolo poco suceptible a la humedad y eficaz contra la penetración del agua proveniente de la precipitación.

- Proporciona unión y cohesión entre agregados, capaz de resistir la acción mecánica de disgregación producida por las cargas de los vehículos. Igualmente mejora la capacidad portante de la estructura, permitiendo disminuir su espesor.[1]

La mezcla asfáltica típica utilizada en Costa Rica está constituida de $95 \%$ de material pétreo y un $5 \%$ de asfalto. Esta es una mezcla que presenta cierta elasticidad y condiciones de recuperación que permite absorber las cargas del tránsito para mitigar las deformaciones. Por otra parte la mezcla es capaz de resistir condiciones severas de temperatura desde bajas hasta altas temperaturas siendo las altas las que aumentan la susceptibilidad a la deformación permanente.

Sin embargo, es muy probable que se acumule deformación plástica debido al servicio, cuando es sometida especialmente a una aplicación repetida de cargas y a temperaturas altas, sobre todo si se trata de vehículos pesados circulando a baja velocidad en climas calientes, como el caso de la Ruta Nacional 1 en el tramo ubicado en Guanacaste o en otras zonas cálidas del país.

Debido a lo anterior, se convierte en una opción mejorar las propiedades reológicas del asfalto modificándolo con la adición de polímeros de tipo elastómeros o plastómeros, en concentraciones entre el $1,5 \%$ y $5 \% \mathrm{~m} / \mathrm{m}$, dependiendo del grado de desempeño requerido y las especificaciones indicadas por el fabricante del polímero. Los polímeros mejoran el desempeño de la mezcla, aumentando la resistencia a la deformación permanente, la resistencia a la fatiga, al mismo tiempo que aumenta su durabilidad al disminuir la oxidación y el deterioro por permeabilidad.
Considerando que la mezcla asfáltica requiere ser homogénea durante todo su proceso productivo, es muy importante incluir en el control de calidad las características físico-químicas del asfalto, pero poco se ha trabajado para determinar la concentración real de polímero en el asfalto una vez modificado, solo se cuenta con la formulación utilizada para su mezcla.

Existen esfuerzos importantes para lograr cuantificar el contenido de polímero, una opción interesante pero requiere de equipo instrumental costoso es mediante Espectroscopía Infrarroja con trasformada de Fourier (FTIR), otra opción es mediante Cromatografía de Permeación en Gel con un Detector de Índice de Refracción (GPC-RID), otra posibilidad es la metodología propuesta por la Unidad de Materiales y Pavimentos del LanammeUCR, que consiste en una variación del método PAT (Particle Additive Test, por sus siglas en inglés) propuesto por el Dr Hussain Bahia y sus colaboradores.

A continuación se presenta una descripción de estos métodos y cómo interpretar los resultados.

\section{Muestra}

Es muy importante considerar que la muestra del ligante asfáltico debe ser representativa, y de ser almacenada, el recipiente debe ser adecuado y las condiciones de almacenamiento óptimas, para evitar contaminación o alteración de las propiedades de la muestra. [1]

La muestra de asfalto modificado debe considerarse homogénea, debido a que las metodologías de análisis podrían generar resultados poco precisos si las muestras presentan diferencias significativas de concentración del polímero a lo largo de la matriz asfáltica. Ver figura 1.

Los polímeros deben de encontrarse en buen estado, sin contaminaciones ni evidencia de alteraciones estructurales del material.

El objetivo de modificar un asfalto con polímero es obtener mejoras en las propiedades reológicas, y por consiguiente en la mezcla asfáltica, la homogeneidad en la incorporación del polímero es muy importante y un control de calidad reológico es una buena técnica para demostrar la homogeneidad y que se modificaron las propiedades lo suficiente para obtener los resultados finales deseados, desde la óptica del desempeño del asfalto. 


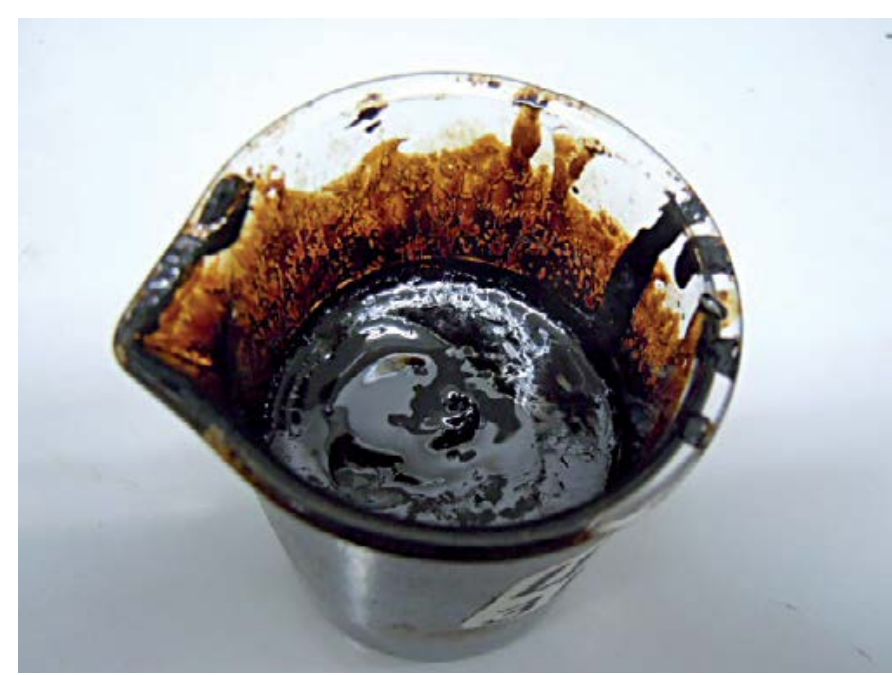

Figura 1. Fotografía de un asfalto modificado con polímero tipo SBS, la imagen muestra un caso de mala incorporación del polímero (presencia de grumos) en la matriz asfáltica.

\section{Análisis de ligantes asfálticos por FTIR.}

\subsection{Determinación cualitativa de grupos funcionales presentes en el ligante asfáltico.}

Hay mucho interés por identificar de forma cualitativa los grupos funcionales presentes en el ligante asfáltico por FTIR (Espectroscopía Infrarroja por transformada de Fourier), ya que permite la identificación molecular de los diferentes grupos funcionales a través de vibraciones moleculares de cada sección de la estructura

Tabla 1. Bandas características de asignación para vibraciones moleculares para los polímeros de clase 1.1.1, los rangos de frecuencia son las especificas para el polímero 2,6,10,15,19,23-hexametil-tetracosano. [10]

\begin{tabular}{|c|c|c|c|c|}
\hline \multirow{2}{*}{$\begin{array}{l}\text { Clase } \\
\text { polímero }\end{array}$} & \multirow{2}{*}{$\begin{array}{l}\text { Rango de } \\
\text { frecuencia } \\
\left(\mathrm{cm}^{-1}\right)\end{array}$} & \multicolumn{2}{|c|}{ Intensidad Relativa } & \multirow{2}{*}{ Asignación } \\
\hline & & FTIR & Raman & \\
\hline \multirow{7}{*}{1.1 .1} & $2950-2970$ & $\mathrm{~s}$ & $\mathrm{~m}$ & $\begin{array}{c}\text { Estiramiento asimétrico } \\
\text { banda } \mathrm{CH}_{3} \text { alifatico }\end{array}$ \\
\hline & 2920 - 2935 & $\mathrm{~s}$ & $\mathrm{~m}$ & $\begin{array}{l}\text { Estiramiento asimétrico } \\
\text { banda } \mathrm{CH}_{3} \text { alifatico }\end{array}$ \\
\hline & $2860-2880$ & $\mathrm{~m}$ & s & $\begin{array}{l}\text { Estiramiento simétrico } \\
\text { banda } \mathrm{CH}_{3} \text { alifatico }\end{array}$ \\
\hline & $2840-2860$ & $\mathrm{~m}$ & s & $\begin{array}{c}\text { Estiramiento simétrico } \\
\text { banda } \mathrm{CH}_{3} \text { alifatico }\end{array}$ \\
\hline & $1450-1470$ & $\mathrm{~m}$ & $\mathrm{~m}$ & $\begin{array}{l}\text { Flexión alifatica bandas } \\
\qquad \mathrm{CH}_{2} \text { y } \mathrm{CH}_{3}\end{array}$ \\
\hline & $\sim 1380$ & $s-m$ & $\mathrm{~m}$ & Flexión $\mathrm{CH}_{3}$ \\
\hline & $720-770$ & $s-m$ & 0 & Balanceo $\mathrm{CH}_{2}$ \\
\hline
\end{tabular}

En la tabla 1 se encuentra el análisis de las bandas FTIR y RAMAN obtenidas para un polímero simple de clase 1.1.1, es importante resaltar que la espectrocopía RAMAN es complementaria del FTIR, en la figura 2 se encuentra el espectro FTIR y RAMAN con la estructura química específica para este polímero.

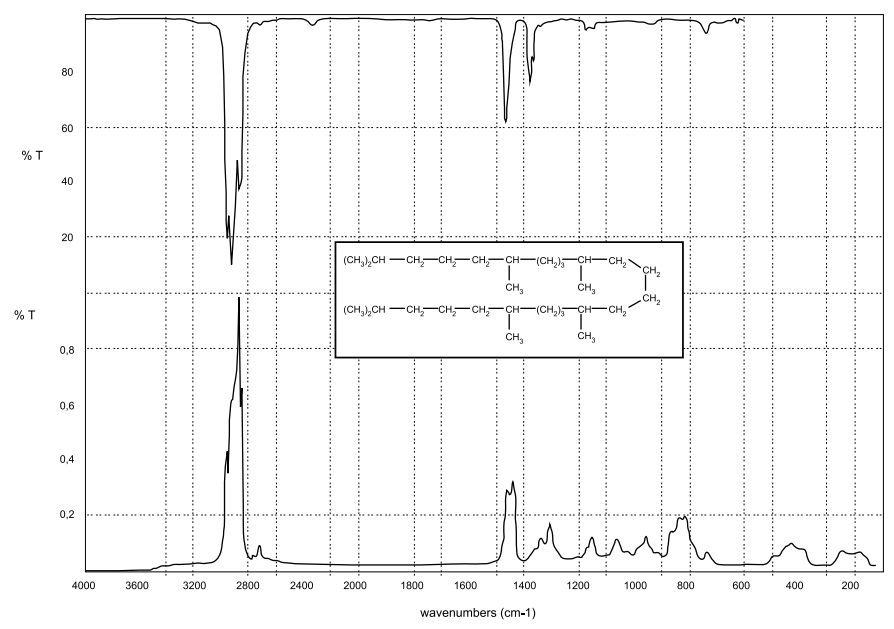

Figura 2. Espectro FTIR (arriba) y RAMAN (abajo) del polímero 2,6,10,15,19,23-hexametil-tetracosano, en la tabla 1 se encuentran sus bandas características que lo identifican y diferencian de los otros compuestos. [10]

En la tabla 2 se analiza de la misma forma el ligante asfáltico patrón interino del LanammeUCR, de igual forma en la figura 3 se encuentra el espectro FTIR para este asfalto.

Tabla 2. Bandas características de asignación para vibraciones moleculares para el ligante asfáltico clasificado como AC-30 (LPI IV).

\begin{tabular}{|c|c|c|}
\hline $\begin{array}{c}\text { Rango de } \\
\text { frecuencia } \\
\left(\mathrm{cm}^{-1}\right)\end{array}$ & $\begin{array}{c}\text { Intensidad } \\
\text { Relativa } \\
\text { FTIR }\end{array}$ & Asignación \\
\hline $2920-2851$ & alta & $\begin{array}{c}\text { Baja de intensidad alta del grupo } \mathrm{CH} 2 \mathrm{y} \\
\mathrm{CH} \text { H Hidrocarbonos saturados }\end{array}$ \\
\hline 1600 & $\mathrm{~m}$ & $\begin{array}{c}\text { Baja de intensidad media de los } \mathrm{C}=\mathrm{C}(\mathrm{Ar}) \\
\text { Hidrocarbonos aromáticos }\end{array}$ \\
\hline 1500 & $\mathrm{~s}$ & $\begin{array}{c}\text { Baja de intensidad baja de los } \mathrm{C}=\mathrm{C}(\mathrm{Ar}) \\
\text { Hidrocarbonos aromáticos }\end{array}$ \\
\hline 1340 & $\mathrm{~m}$ & $\begin{array}{c}\text { Baja de intensidad media de los } \mathrm{C}-\mathrm{N}-\mathrm{H} \\
\text { Aminas aromáticas }\end{array}$ \\
\hline 1170 & $\mathrm{~s}$ & $\begin{array}{c}\text { Baja de intensidad baja de los } \mathrm{R}-\mathrm{O}-\mathrm{Ar} \text { de } \\
\text { los alquil Aril eteres }\end{array}$ \\
\hline 1030 & $\mathrm{~s}$ & $\begin{array}{c}\text { Baja de intensidad baja de los } \mathrm{R}-\mathrm{O}-\mathrm{Ar} \text { de } \\
\text { los alquil Aril eteres }\end{array}$ \\
\hline
\end{tabular}




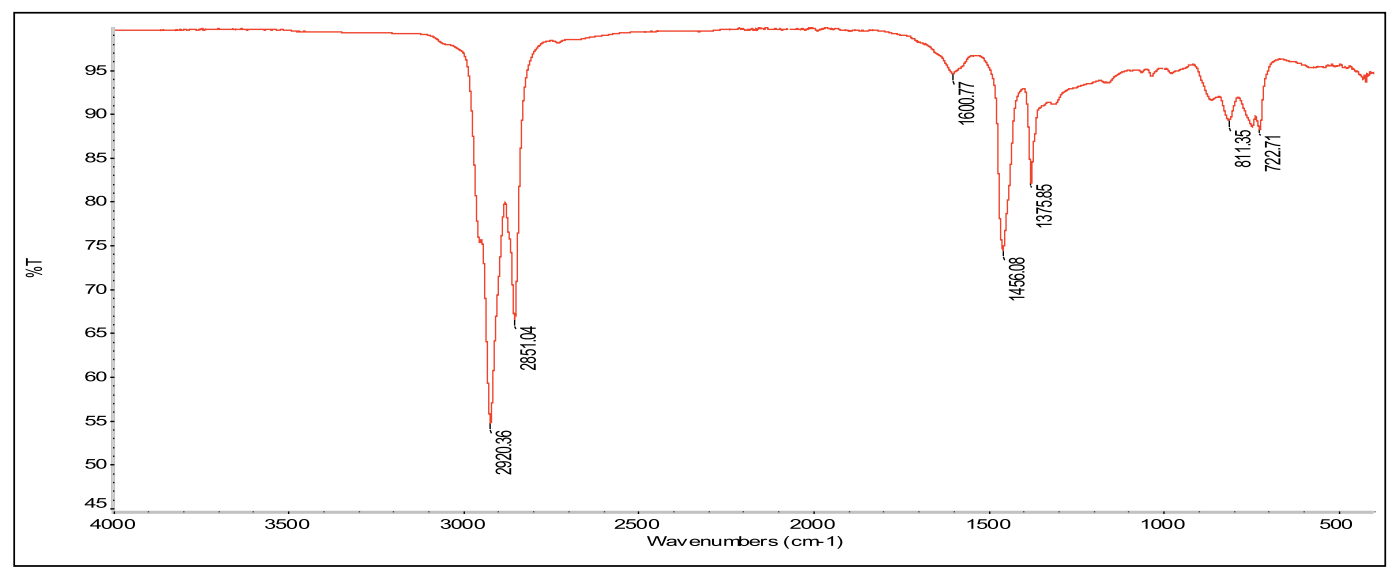

Figura 3. Espectro FTIR de un asfalto clasificado como AC-30 (LPI IV), se muestra la identificación de las bandas de vibración molecular típicas en la tabla №2.

\subsection{Determinación cuantitativa del contenido de polímero en el ligante asfáltico mediante FTIR (curva de calibración).}

Independiente del método de FTIR a utilizar para determinar el contenido de polímero en asfalto modificado, es indispensable la generación de la curva de calibración que permitirá determinar el contenido de polímero en la muestra desconocida. Para esto se producen patrones de concentración conocida y se determinan los espectros IR.

Se producen asfaltos modificados con un polímero determinado en concentraciones de $0 \%, 1 \%, 1,5 \%, 2 \%$, $2,5 \%, 3 \%$ y $5 \% \mathrm{~m} / \mathrm{m}$. Se obtienen los espectros IR y mediante un software de integración, se genera una curva por "zonas" donde al menos tres bandas del IR que muestren evidencia de cambio en el espectro serán las consideradas para la integración.

Una vez obtenido un coeficiente de correlación aceptable para el método, se evalúa la muestra desconocida bajo la misma metodología que los patrones de la curva y el software indicara el contenido en $\% \mathrm{~m} / \mathrm{m}$ de polímero que la muestra contiene. De esta forma se obtendrá cuantitativamente el porcentaje de polímero incorporado en el ligante asfalto en la muestra medida.

En la figura 4 se muestra un ejemplo de los espectros FTIR para una curva de calibración de un aceite de referencia para viscosidad $\mathbf{S 6 0}$, en diferentes concentraciones para este caso se utiliza los espectros en modo de absorbancia y en la figura 5 la curva de calibración final para la determinación cuantitativa y puede ser avaluada cualquier muestra del $\mathbf{S 6 0}$ de concentración desconocida.

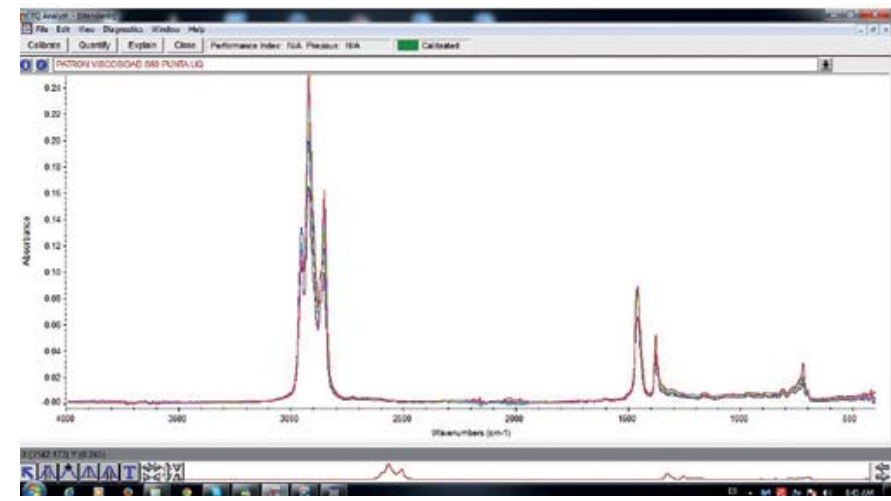

Figura 4. Espectros FTIR de muestras para la curva de calibración en modalidad de absorbancia, para el aceite de referencia $\mathrm{S} 60$, las diferencias de intensidad son debidas a las diferentes concentraciones de los patrones.

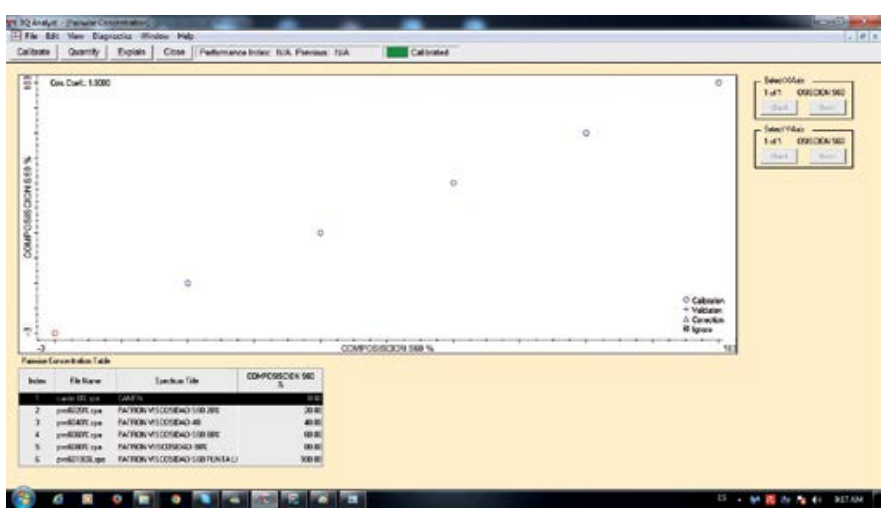

Figura 5. Curva de calibración obtenida para los deferentes patrones del aceite de referencia S60 por FTIR, según la ley de Beer-LambertBouguer. 


\subsection{Determinación del contenido de polímero en el ligante asfáltico mediante FTIR (Método de Placa de sal).}

Otro método utilizado para la determinación cuantitativa del contenido de polímero en ligantes asfálticos es mediante deposición de la muestra en una placa de cristal transparente en el IR, normalmente de bromuro de potasio, bromuro de sodio o cloruro de sodio. [5]

Como se indica en el punto 3.2 debe existir una curva de calibración y un sistema de interpretación de datos para determinar la concentración de polímero en la muestra desconocida. En la figura 6 se muestra la placa de cristal y su ubicación en el paso óptico del equipo.
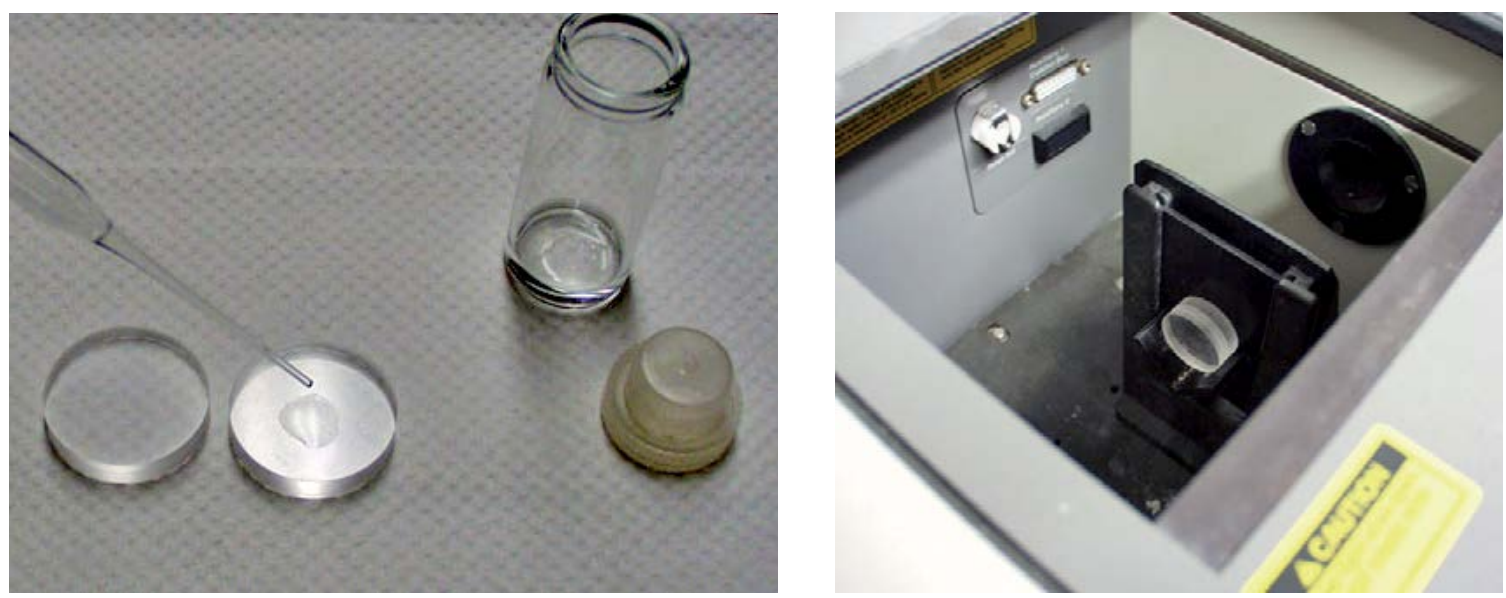

Figura 6. Detalle del método de placa de sal, incorporación de la muestra (imagen izquierda), placa de sal colocada en el paso óptico del FTIR. [11]

\subsection{Determinación del contenido de polímero en ligantes asfálticos mediante FTIR (Método utilizando ATR).}

Esta metodología ha solucionado muchos de los problemas que se tenían con las muestras líquidas y sólidas ya que no se requiere generar pastillas de Bromuro de potasio o placas salinas, el sistema ATR de las siglas en inglés Attenuated Total Reflectance (reflectancia total atenuada), es una técnica en FTIR que permite medir muestras de forma directa sobre la superficie de un cristal de diamante o germanio obteniéndose espectros de forma muy rápida y con muy buena resolución. [5-6]

La muestra se coloca sobre la superficie de la celda de cristal y se mide de forma directa, el espectro aparece segundos después en la pantalla del software.

Como se indica en el punto 3.2 debe existir una curva de calibración para determinar la concentración de polímero en la muestra desconocida. En la figura 7 se muestra el sistema ATR obteniendo un espectro Infrarrojo de un ligante asfáltico.
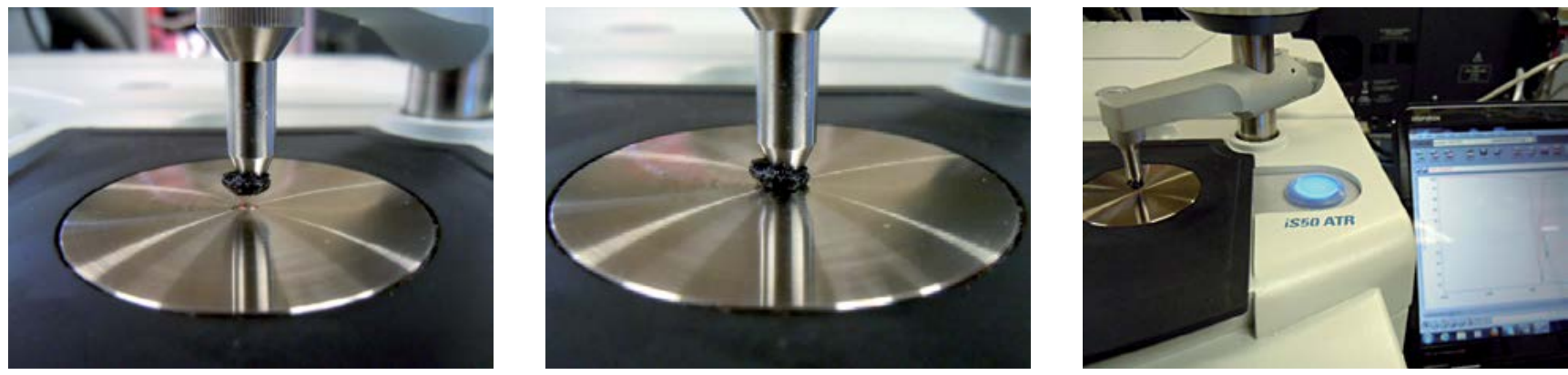

Figura 7. Detalle del método de FTIR-ATR, sistema con cristal de diamante (imagen a la izquierda), colocación de muestra en el cristal (imagen al centro) y determinación del espectro de la muestra de ligante asfáltico (imagen a la derecha). 


\subsection{Determinación del contenido de polímero en ligantes asfálticos mediante FTIR (Métodos clásicos).}

Dos métodos figuran como técnicas clásicas, una es producir pastillas de bromuro de potasio con la muestra, o medir la muestra en una celda de flujo ambas en modo de transmitancia.

En el primer caso se pulveriza bromuro de potasio y se adiciona la muestra de interés se coloca la mezcla final en un generador de pastillas, se genera una pastilla casi trasparente para colocar en la paso óptico del IR, la principal desventaja de este método es que la calidad del resultado final depende por mucho de la generación y trasparencia de la pastilla de $\mathrm{KBr}$ con la muestra.
El segundo caso es producir una disolución del material desconocido y un solvente, generalmente Tetrahidrofurano y medir en la celda de flujo, para este caso hay que considerar las bandas del IR del solvente utilizado que no corresponden a la muestra de interés.

De la misma forma hay que hacer una curva de calibración para cada polímero, y para estos dos métodos en particular puede ser muy difícil de alcanzar resultados con buena precisión. Los resultados dependerán mucho de la destreza del analista y bajo esta condición se recomienda mejor la técnica por ATR. En la figura 8 se muestra el sistema de paso óptico para pastillas de bromuro de potasio y un ejemplo de una celda de flujo típica para un FTIR.
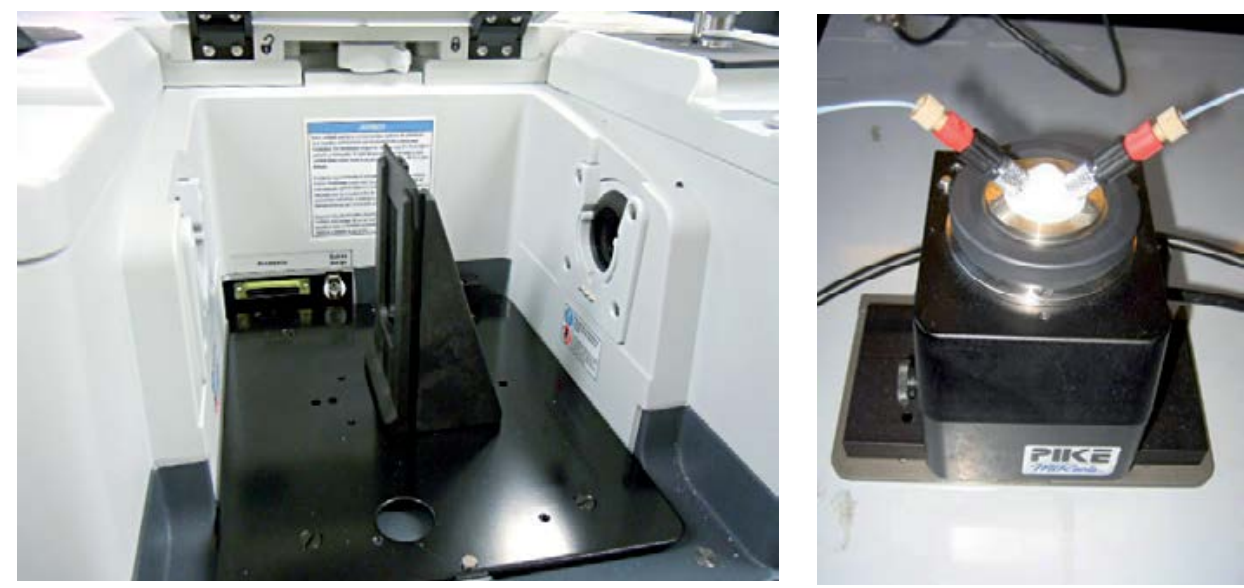

Figura 8. Detalle del equipo de FTIR-KBr, sistema con pastillas de bromuro de potasio en sistema de paso óptico clásico (imagen a la izquierda), imagen del dispositivo de flujo para muestras en disolución (imagen a la derecha). [12]

\section{Análisis de ligantes asfálticos modificados con polímeros por GPC-RID.}

La técnica GPC-RID de las siglas en inglés de "Gel Permeation Chromatography", es una técnica muy utilizada para separar materiales en una solución por su tamaño molecular mediante un RID Refractive Index Detector, de forma general la muestra se disuelve un solvente apropiado y se inyecta en el GPC este separa mediante tiempo de retención, donde las estructuras de las moléculas más pesadas migraran de forma más lento y llegaran al detector de índice de refracción de ultimo obteniendo un cromatograma.

Este cromatograma puede ser interpretado integrando el área bajo la curva y de esta manera comparar una muestra desconocida con resultados obtenidos en la curva de calibración.[7]

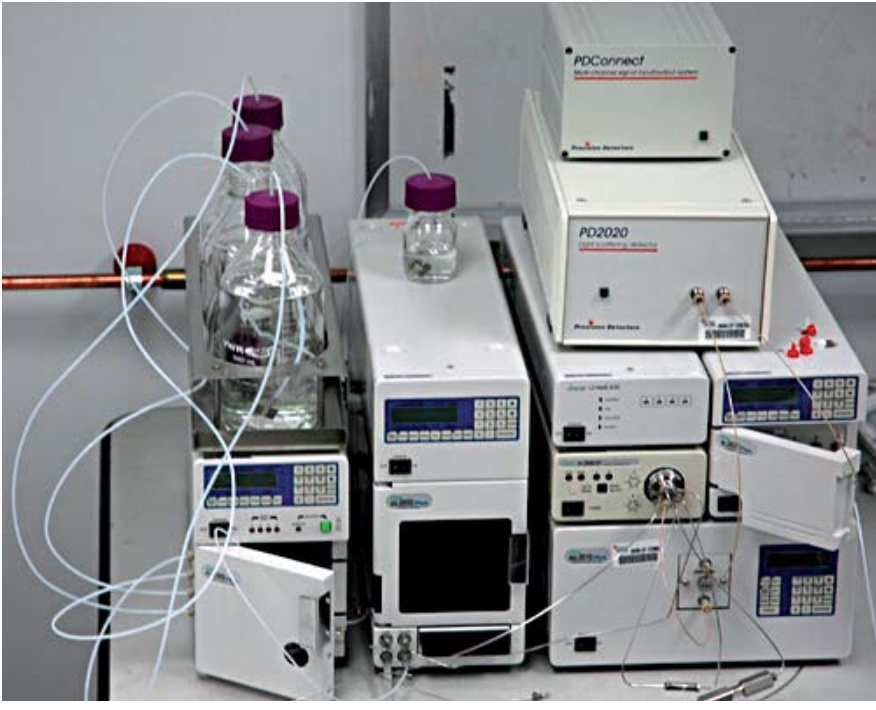

Figura 9. Detalle del equipo de GPC, la metodología para determinar el contenido de polímeros en ligantes asfálticos requiere adicional un detector de índice de refracción RID. [13] 


\section{Análisis de contenido de polímero} mediante el procedimiento para el análisis de partículas de aditivo (PAT) modificado.

Este método de ensayo se basa en el método propuesto por el Dr Hussain Bahia y sus colaboradores, para determinar de forma cualitativa la presencia de partículas poliméricas, pero se modifica para lograr no solo la identificación de la presencia del polímero, sino también cuantificar la cantidad de polímero SBR adicionado.

El alcance de este método es recuperar polímeros del tipo SBR y el polímero recuperado puede ser identificado mediante FTIR para su identificación cualitativa, y mediante diferencia de masa, cuantificarse. Este método en una herramienta sencilla de control de calidad de cantidad de polímero adicionado al asfalto en planta. [8-9]

Una muestra de ligante asfáltico se disuelve en n-hexano, la disolución final se filtra en el equipo PAT, el residuo retenido en la malla (asfaltenos y polímero) se lava con diclorometano, el residuo final presente en la malla es el polímero retenido, por diferencia de masa se obtiene el porcentaje de polímero presente en la muestra. El residuo presente indica la presencia de polímero tipo SBR (el ligante asfáltico sin modificar no deja residuo ni el ligante asfáltico modificado con SBS). El equipo PAT se muestra en la figura 10 y 11.
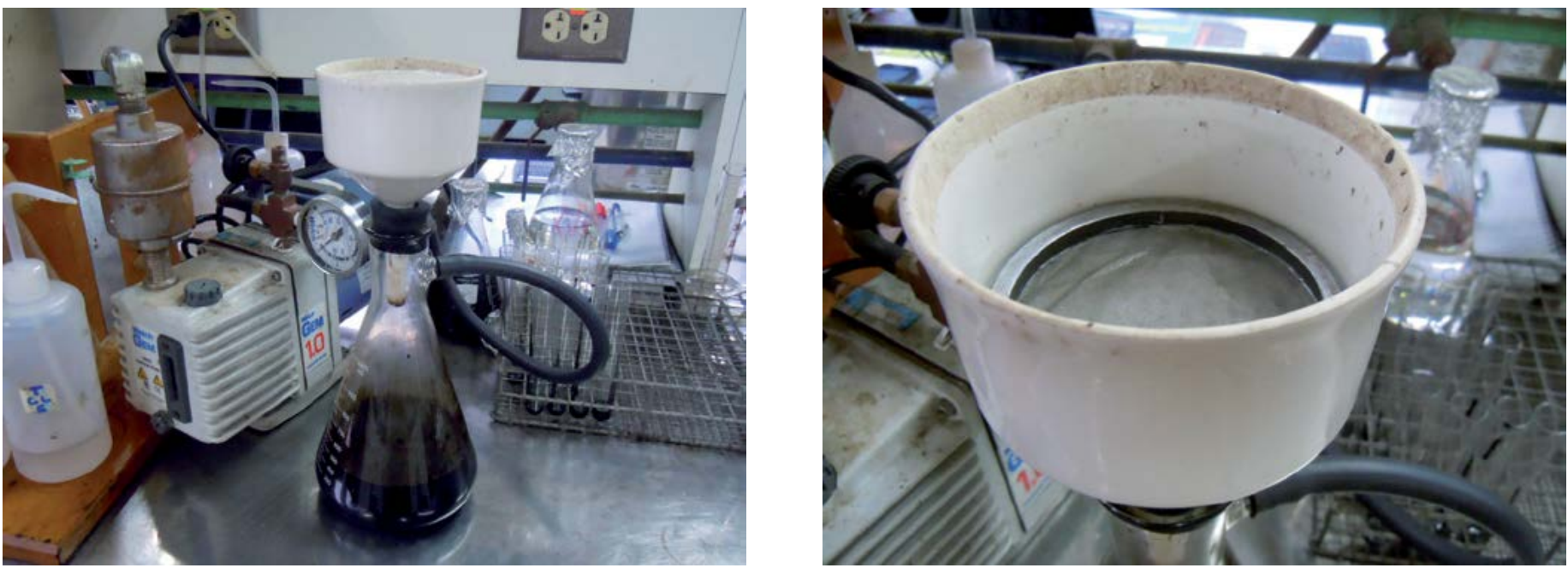

Figura 10. Detalle del equipo de PAT modificado, para determinar el contenido de polímero después de disuelto (imagen izquierda), colocación de la malla de $75 \mu \mathrm{m}$ en el embudo Buhner.
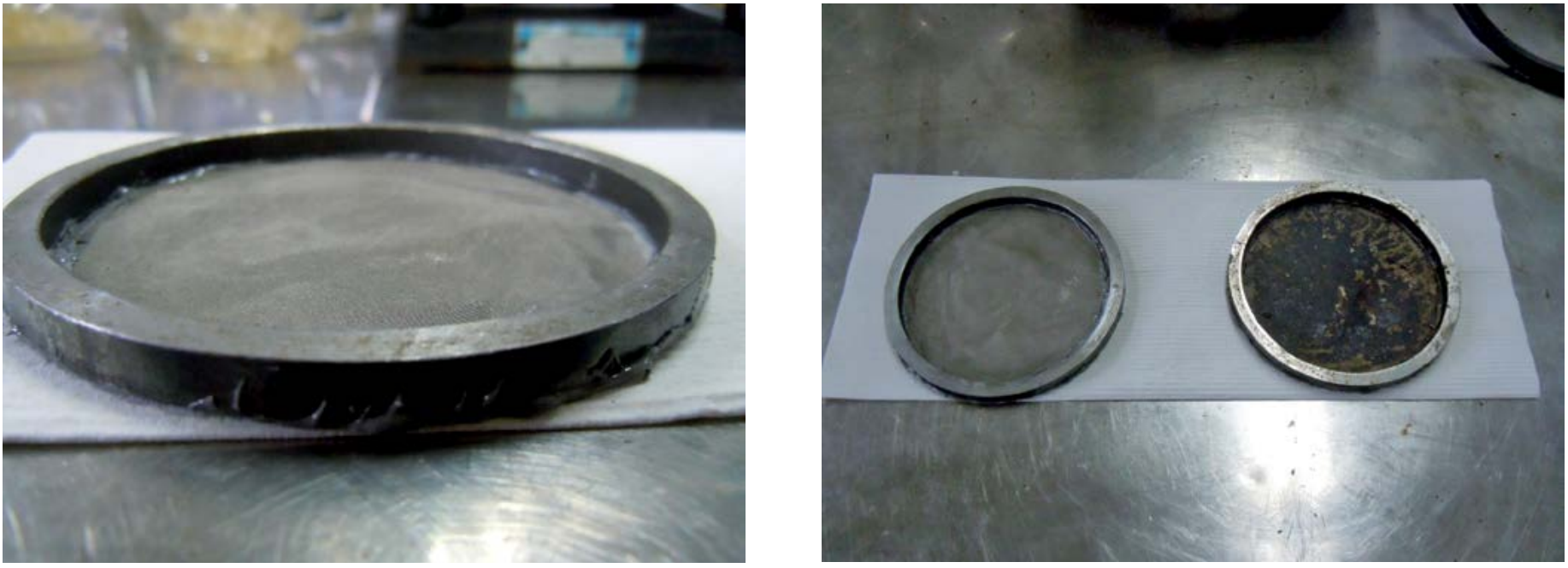

Figura 11.Detalle de la malla de $75 \mu \mathrm{m}$ o malla $N^{\circ} 200$ (imagen izquierda), recuperación de polímero tipo SBR en la malla (imagen derecha) 


\section{Recomendaciones}

Este documento es una guía de los ensayos que se pueden realizar para un apropiado control de calidad del contenido de polímero incorporado al ligante asfáltico, es importante considerar que este se encuentra cerca del $2 \%$ al $5 \% \mathrm{~m} / \mathrm{m}$ en el ligante asfáltico modificado, es relativamente bajo y solo de esta forma se puede asegurar la calidad del proceso de modificación de asfalto y su utilidad como insumo para la construcción de carreteras con pavimento asfáltico.

Considerando los métodos presentados en este documento para caracterizar los materiales bituminosos modificados se realizan las siguientes recomendaciones:

- El polímero debe ser incorporado de forma adecuada a la matriz asfáltica.

- Es necesario asegurarse que la muestra de ensayo sea tomada y almacenada de forma apropiada, ya que dependiendo esto influirá en los resultados finales de ensayo.

- Si no se cuenta con equipo instrumental, se podría implementar la cuantificación mediante el equipo del PAT modificado. Es de muy bajo costo y fácil de implementar.

- La técnica de FTIR permite la cuantificación mediante software de integración que es costoso, pero muy preciso, también la identificación cualitativa puede funcionar como herramienta simple de control de calidad evidenciando presencia de polímero cuando se mide la muestra al ser comparada contra una de referencia.

- La cromatografía de permeación en gel (GPC-RID), es novedosa y costosa, si se cuenta con este equipo se puede cuantificar de esta forma, si se considera en inversión se recomienda evaluar todas las opciones en el mercado.

- Cuantificar por los métodos clásicos de IR, no se recomienda debido a su poca precisión y a las variaciones que puede generar el analista durante las lecturas.

- Los resultados de ensayo deben realizarse mínimo por duplicado o triplicado e incluir la precisión del resultado.

- Los resultados de carácter reológico son muy susceptibles a la temperatura por lo que se recomienda cuidados especiales en el tratamiento térmico previo de la muestra.

\section{Referencias}

1. Métodos \& Materiales. Vol. 1, $N^{\circ} 1,2011$

2. Jour.Chem.Soc.Pak. Vol.29, N 2007.

3. Secretaria General de la Organización de los Estados Americanos, Programa Regional de Desarrollo Científico y tecnológico, Espectroscopia Infrarroja, Serie Química, Monografía N¹2, 1981.

4. K. Nakanishi, Infrared Absorption Spectroscopy, Second Edition, Holden-Day Inc, 1977.

5. Texas Department of Transportation, TxDOT, Tex-533C, Test procedure for Determining Polymer Additive Percentages in Polymer Modified Asphalt Cements", 8 páginas, Estados Unidos, 1999.

6. American Association of State Highway and Transportation Officials. AASHTO T302, Polymer Contend of Polymer-Modified Emulsified Asphalt Residue and Asphalt Binders, 6 páginas, Estados Unidos, 2012.

7. American Association of State Highway and Transportation Officials. AASHTO Txxx-xx, Determination of Polymer Modifier in Asphalt, Draft , 16 páginas, Estados Unidos, 2005.

8. National Cooperative Highway Research Program, NCHRP, "Report 459 Characterization of Modified Asphalt Binders in SuperPave Mix Design", Appendix I Standard Test Method for Determining the Content of Solid Additives in Asphalt Binder Using the Particulate Additive Test (PAT) Device, C-1-1 a C-1-4, 2001.

9. Federal Highway Administration, FHWA, U.S. Department of Transportation, Evaluation of the Particulate Additive Test, Publication NO. FHWA-HRT-04-110, 32 pages, 2004.

10. A.H. Kuptsov; G.N. Zhizhin, Handbook of Fourier Transform Raman and Infrared Spectra of polymers, Elsevier, 1998, pp-XXIII-XXV, 5.

11. ACP Science for life, IR Spectroscopy, Experimental procedure, salt plate method images, http://www.organicchem.org/oc1web/exp/ir/ irpro.html

12. Working group on process Analysis \& Vibrational Spectroscopy, Equipment http://www.iac.tuwien.ac.at/cavs/equipment.php

13. University of Central Florida, NanoScience Technology Center, Gel Permeation Chromatography, http://www.nanoscience.ucf.edu/ equipment/details.php?id=7 